\title{
Development, promotion, and application of online OvAge calculator based on the WeChat applet: Clinical prediction model research
}

\section{Wenwen Xu}

Jiangsu Provincial Hospital of Traditional Chinese Medicine, Affiliated Hospital of Nanjing University of Chinese Medicine

\section{Hui Wang}

Nanjing Medical University

LinTing Han

Nanjing Medical University

\section{Xueli Zhao}

Nanjing Medical University

\section{PanPan Chen}

Nanjing Medical University

\section{Haiyang Zhao}

Nanjing Medical University

Jing Jin

Jiangsu Provincial Hospital of Traditional Chinese Medicine, Affiliated Hospital of Nanjing University of Chinese Medicine

\section{Zheng Zhu}

Nanjing Medical University

Qingling Ren ( $\sim$ yfy0047@njucm.edu.cn)

Jiangsu Provincial Hospital of Traditional Chinese Medicine, Affiliated Hospital of Nanjing University of Chinese Medicine

\section{Fang Shao}

Nanjing Medical University

\section{Research Article}

Keywords: Ovarian age, Subject recruitment, WeChat applet, Model optimization

Posted Date: March 3rd, 2022

DOl: https://doi.org/10.21203/rs.3.rs-1290408/v2 
License: (c) (i) This work is licensed under a Creative Commons Attribution 4.0 International License. Read Full License 


\section{Abstract}

Background: Currently, there are many clinical markers and models for evaluating ovarian reserve. However, none of them is ideal, especially the ovarian reserve assessment model applied to Chinese women, which needs further research. It is well known that the number of clinical samples is the main factor limiting the specificity and sensitivity of markers and models. At the same time, the traditional method of recruiting subjects requires a lot of time and has a high cost.

Purpose: In this study, to explore the possibility of subjects self-reporting through WeChat applets and to provide more data for further optimization of the OvAge model, we developed an ovarian age prediction model for Chinese women.

Methods: This study developed an online Ovarian Age区OvAge区 calculator based on the WeChat applet and applied a generalized linear model to optimize the ovarian age prediction model that meets the physiological characteristics of Chinese women.

Results: Compared with traditional recruitment methods, the online OvAge calculator can collect a large number of subjects in a very short time, which is efficient and convenient. In addition, the optimized formula, OvAge $=\exp (3.5254-0.001 * \mathrm{PRL}-0.0231 * \mathrm{AMH})$, is currently a suitable model for ovarian age assessment of healthy Chinese women. We applied the final equation to the healthy population, decreased ovarian reserve (DOR), and polycystic ovary syndrome (PCOS) population datasets. The results show that there is no significant difference between ovarian age and chronological age in healthy people, and the prediction accuracy is high.

Conclusion: The OvAge calculator based on the WeChat applet is a new type of online self-report system that can collect a large number of subjects in a short time and can continuously optimize the model and update the applet version. This model is economical and efficient, and it is worthy of [1]promotion. The optimized OvAge model also provides a reference value for the clinical evaluation of ovarian reserve.

\section{Introduction}

In reproductive medicine, a woman's fertility potential can be used to measure the likelihood of a successful pregnancy using assisted reproductive technology. Domestic and foreign scholars in the field of reproduction have proposed models of female ovarian scoring and staging systems for predicting pregnancy outcomes, such as What's My Fertility, Stages of Reproductive Aging Workshop (STRAW), OvAge, and the AFA (anti-Müllerian) Hormone level-follicle-stimulating hormone level-age) models ${ }^{1-5}$. These models can help women of childbearing age to effectively understand their fertility status so that they can reasonably plan for pregnancy. Due to confounding factors such as age, genetics, environment, psychology, society, lifestyle, iatrogenic infection, immunity, etc., the age of the ovaries of women of the same chronological age is also very different ${ }^{6-11}$. In addition, many documents show that the reproductive potential of women gradually decreases with age. With the implementation of China's multiple birth policy 
and the increase in the number of older women, it is essential to accurately predict the relationship between chronological age and ovarian age, assess fertility potential, and predict the time of menopause.

Currently, clinical and academic indicators include chronological age, anti-Muller hormone (AMH), antral follicle count (AFC), follicle-stimulating hormone (FSH), clomiphene citrate stimulation test (CCST), inhibin $\mathrm{B}$ (INHB), among others, or a combination of the above indicators to obtain a scoring system and staging model to predict ovarian age and pregnancy outcomes ${ }^{12-16}$. The ideal scoring system and model should be universally accepted, repeatable, easy to understand, and applicable to the general population. In addition, the model can more intuitively predict fertility, menopausal time, reproductive prognosis, and the success rate of assisted reproductive technology.

With the rapid development of the internet and 5G, people's lives are inseparable from the internet. In the current open network environment, WeChat has become a phenomenon-level application software in China ${ }^{17}$. Therefore, we combined the existing OvAge model to properly optimize and develop an online OvAge calculator based on the WeChat applet. To obtain the estimated value of ovarian age, the subject could directly search for the "ovarian age calculator" applet in WeChat and then enter the test interface. The WeChat applet is divided into a basic version (to understand the age of the ovaries, subjects need to provide 2-4 days of menstrual test data, including 6 sex hormone tests, AMH, and AFC tests) and an advanced version (to understand ovarian age and fertility assessment, subjects need to provide 2-4 days of menstrual test data, including 6 sex hormone tests, AMH test, AFC test, and transvaginal ultrasound). Subjects only need to enter the required values in the applet to obtain their predicted ovarian age or ovarian age and fertility assessment results and obtain professional medical advice. The new method of collecting basic data from Chinese women and gynecological examination reports through the online OvAge calculator will explore the possibility of providing subjects with a self-report system through the WeChat applet and provide more data support for the further optimization of the online OvAge calculator.

\section{Materials And Methods Choice of indicators}

In data collection for this study, we collected the $A M H, F S H$, luteinizing hormone $(\mathrm{LH})$, estradiol $\left(\mathrm{E}_{2}\right)$, progesterone $(P)$, testosterone $(T)$, prolactin (PRL), and vitamin D levels of subjects from Day 2 to Day 4 of the menstrual cycle. At the same time, we collected pelvic ultrasound images on the same day of hormone testing and recorded the subjects' height and weight.

\section{Subject's data collection}

This process is divided into three stages: (1) Phase I data collection from May 1 to June 30, 2020. We collected clinical data from subjects from the Department of Gynecology and Reproductive Medicine of Jiangsu Provincial Hospital of Traditional Chinese Medicine, including health, PCOS, and DOR populations. Within two months, we recruited 113 women (16 healthy individuals, 45 PCOS, and 52 DOR populations). The study was approved by the China Clinical Trials Registry (Registration Number ChiCTR2000037522) and the Medical Ethics Committee of Jiangsu Hospital of Traditional Chinese Medicine (Application 
Number 2019NL-152-02). The experimental design fully considered the principles of safety and fairness, and the study did not cause any harm or risk to the subjects. Recruitment was based entirely on the principle of voluntary and informed consent, and the privacy of volunteers was protected as much as possible. In addition, there were no conflicts of interest in this study, (2) Phase II data collection occurred from July 1 st to August 31 st, 2020 , and the subjects were recruited through a contract research organization (CRO) company for a fee. A total of 52 healthy women were included. All tests were carried out in the laboratory of Jiangsu Provincial Hospital of Traditional Chinese Medicine,(3) Phase III data collection occurred due to the low collection efficiency in the first two stages. We tried a brand new online OvAge calculator with a subject self-report system based on a WeChat applet. We clarified the functions and requirements of the OvAge calculator and handed it over to a professional software development company to complete using JavaScript and WXML (WeiXin markup language) development languages, and the basic software version 1.0.0 launched on September 23rd, 2020. In two weeks, more than 9,000 sample data points were collected using the online OvAge calculator in the WeChat applet. After screening, 97 cases of healthy population data were available.

\section{Subjects}

The age range of patients participating in this study through the above three recruitment methods was 1555 years old.

The training set object used to build the model was healthy women. The inclusion criteria for healthy women were a history of spontaneous conception, intact ovaries, and normal menstruation, with an average interval of 21 to 35 days. The exclusion criteria were estrogen or progesterone use or breastfeeding two months before registration, pregnancy, history of female infertility, endometriosis, ovarian cystic mass, history of ovarian surgery, PCOS, gynecological malignancy, history of radiotherapy or chemotherapy, autoimmune diseases, chronic systemic metabolic and endocrine diseases including hyperandrogenemia, hyperprolactinemia, diabetes, thyroid disease, hypogonadotropic hypogonadism, and drug usage that may cause irregular menstruation.

In addition, the test set also included PCOS and DOR populations. PCOS was diagnosed according to Rotterdam criteria, and there were at least 2 of the following 3 characteristics: hypomenorrhea/amenorrhea, clinical and/or biochemical hyperandrogenemia, and polycystic ovarian morphology (PCOM) ${ }^{18}$. The diagnosis of DOR has not been standardized, and there is no international standard. Combined with the current guidelines of the National ART Surveillance System (NASS) and Society for Assisted Reproductive Technology (SART), the diagnostic criteria used in this study were age $\geq 40$ years old, basic FSH $\geq 12$ $\mathrm{mlU} / \mathrm{mL}, A F C \leq 5 \sim 7$, and $\mathrm{AMH} \leq 1.1 \mathrm{ng} / \mathrm{mL}$. Meeting two or more of the above four items was determined to be DOR ${ }^{19}$.

In this study, subjects from gynecology and reproductive medicine clinics, CRO companies, and OvAge WeChat applets were screened based on the above criteria, and past medical history was collected through professional questionnaires.

\section{Statistical analysis, model construction, and optimization}


R software (version 4.0.2) was used to analyze a dataset of 262 subject records in the first to third phases. The whole analysis consists of 4 main steps (Fig. 1).

(i) First, we divide the data collected in the three stages into three datasets: training set, test set, and prediction set. The training set includes phase II and phase III data, which include 52 and 97 observation data points, respectively. The prediction set included phase I data, including healthy controls (HC, 16 subjects), DOR (52 subjects), and PCOS (45 subjects). We checked each dataset to ensure data quality, including missing values and consistency. For missing data, discard the corresponding observations and then perform modeling.

(ii) To describe the relationship between the new response variable, OvAge, and the set of inputs, which are the independent variables, we applied generalized linear model (GLM) theory to our research for the training set. The GLM provided a unified method for modeling all types of response variables, such as continuous, binary, and ordinal response or variables in the form of proportions. It has been hypothesized that in the healthy population, OvAge is equal to chronological age, which is not continuous and nonnormal.

Therefore, a Poisson distribution was chosen as the random component of the GLM for modeling the expected value of OvAge. Identity and logarithm were chosen as candidate link functions. For Ockham's razor (law of parsimony) and avoiding collinearity problems due to the possible nonindependence of predictor variables, stepwise selection, a conventional variable selection method, was applied using the Akaike information criterion (AIC). We first constructed regression models for only Phase 2 data. Next, we used the same variables in the models for only Phase 2 data to fit corresponding models for both Phase 2 and 3 data.

(iii) Considering all possible interactions among covariates to assess any improvements in AIC value and accuracy, the best model was rebuilt. Interaction terms in the model provide interactive effects and were considered when the main effects were significant. The final model was chosen by comparing pairs of models for only Phase 2 data and both Phase 2 and 3 data with the assessment of prediction indices, mean square error (MSE), root mean squared error (RMSE), and mean absolute error (MAE), for the test set of 19 observations. (iv) The final generalized linear model was applied to the healthy controls (HCs), DOR, and PCOS datasets in the prediction set. The hypothesis was that, in the case of HCs, the ovarian age would be equal to the chronological age. In the case of DOR, the ovarian age would be higher than the chronological age, and in the case of PCOS, the ovarian age would be lower than the chronological age.

\section{Results}

\section{The OvAge calculator in the literature is not fully adapted to the Chinese population}

According to the OvAge calculator in the literature, during phase I data collection in this study, we collected the chronological age of 113 women ( 16 healthy individuals, 45 PCOS, and 52 DOR patients) with five indicators related to the formula (Table 1). 
Table 1

Participant data from Phase I.

\begin{tabular}{|llll|}
\hline Indicators & $\begin{array}{l}\text { Healthy women } \\
(\mathbf{n = 1 6})\end{array}$ & $\begin{array}{l}\text { DOR women } \\
(\mathbf{n}=\mathbf{5 2})\end{array}$ & $\begin{array}{l}\text { PCOS women } \\
(\mathbf{n}=\mathbf{4 5})\end{array}$ \\
\hline Chronological age & $28.44 \pm 2.99$ & $29.27 \pm 3.76$ & $28.33 \pm 2.83$ \\
\hline AMH $(\mathrm{ng} / \mathrm{mL})$ & $3.64 \pm 3.42$ & $1.02 \pm 1.03$ & $12.79 \pm 3.43$ \\
\hline FSH $(\mathrm{mlU} / \mathrm{mL})$ & $6.28 \pm 2.00$ & $22.70 \pm 11.64$ & $5.80 \pm 1.87$ \\
\hline PRL $(\mathrm{ng} / \mathrm{mL})$ & $14.28 \pm 5.13$ & $12.43 \pm 4.51$ & $10.72 \pm 4.78$ \\
\hline AFC & $5.63 \pm 2.09$ & $3.42 \pm 1.39$ & $9.14 \pm 4.37$ \\
\hline BMI & $23.39 \pm 4.22$ & $23.36 \pm 2.81$ & $26.58 \pm 6.41$ \\
\hline
\end{tabular}

We used the OvAge calculator in the literature to process the Phase I data and verified their biological age with the predicted ovarian age. The chronological age of PCOS patients was significantly older than the ovarian age calculated by OvAge $(p<0.001)$. The chronological age of DOR patients was significantly younger than the ovarian age calculated by OvAge $(p<0.05)$. There was no significant difference between the chronological age of healthy individuals and the ovarian age calculated by OvAge, but the $P$ value was close to the critical value, suggesting a trend of difference (Table 2). The above results indicate that the chronological age for healthy people is not completely consistent with the ovarian age calculated by OvAge in the literature.

Table 2

Comparison of chronological age and age calculated by OvAge in the literature.

\begin{tabular}{|llll|}
\hline Indicators & chronological age & Literature OvAge & P value \\
\hline Healthy women $(\mathrm{n}=16)$ & $28.44 \pm 2.99$ & $30.63 \pm 3.02$ & 0.0817874 \\
\hline DOR women $(\mathrm{n}=52)$ & $29.27 \pm 3.76$ & $31.29 \pm 5.42$ & 0.0402479 \\
\hline PCOS women $(\mathrm{n}=45)$ & $28.33 \pm 2.83$ & $26.55 \pm 2.46$ & 0.0002038 \\
\hline
\end{tabular}

\section{The establishment of the generalized linear model for the new OvAge calculator}

For healthy people, the chronological age was not exactly the same as the ovarian age calculated by OvAge in the literature, suggesting that this calculator is not completely suitable for Chinese women. To establish a more suitable ovarian age assessment method for the Chinese population, we tried to optimize the OvAge formula. Age was the dependent variable, and the independent variables for modeling were $B M I, P, T, E_{2}$, $\mathrm{PRL}, \mathrm{LH}, \mathrm{FSH}$, and $\mathrm{AMH}$. We considered normal linear regression, a combined generalized linear model with logarithm and identity link functions, and modeled it with the independent variable of age. We used stepwise linear regression based on the AIC value for variable selection and constructed a regression model for the phase II data, which are represented as mod1a, mod2a, and mod3a (Mod1. indicates that the dataset conforms to the normal distribution. Mod2. indicates that the dataset is a nonnormal Poisson 
distribution, and the conventional glm, log-link model is selected. Mod3. indicates that the dataset is a nonnormal Poisson distribution and that the conventional identify-link model is selected. The "a" indicates that the selected dataset is from dataset II, and "b" indicates that the selected dataset is from dataset II and dataset III. "a" indicates that the selected dataset is from dataset II. Mod1a, mod2a, and mod3a only contain PRL and AMH variables. PRL was not statistically significant, whereas AMH was statistically significant (Table 3).

Table 3

Summary of model fit for Phase II.

\begin{tabular}{|llllll|}
\hline item & & Estimate & Std. Error & t value & p value \\
\hline mod1a & (Intercept) & 32.3968 & 1.903 & 17.0244 & 0 \\
& PRL & 0.1483 & 0.0872 & 1.7001 & 0.0955 \\
& & & & & $(-)$ \\
& AMH & -0.8752 & 0.3296 & -2.6557 & 0.0106 \\
& & & & & $\left(^{*}\right)$ \\
mod2a & (Intercept) & 3.4846 & 0.0568 & 61.4003 & 0 \\
\hline & PRL & 0.0043 & 0.0024 & 1.7452 & 0.081 \\
& & & & & $(-)$ \\
\hline & AMH & -0.0287 & 0.0103 & -2.7813 & 0.0054 \\
& & & & & $(* *)$ \\
\hline mod3a & (Intercept) & 32.2573 & 1.7938 & 17.9829 & 0 \\
\hline & PRL & 0.1438 & 0.0867 & 1.6577 & 0.0974 \\
& & & & & $(-)$ \\
\hline & AMH & -0.8163 & 0.2971 & -2.7474 & 0.006 \\
& & & & & $(* *)$ \\
\hline
\end{tabular}

According to the results of prediction errors Mean Square Error (MSE), Root Mean Square Error (RMSE), and Mean Absolute Error (MAE) (Table 4), the smaller the MSE, RMSE, and MAE, the better the prediction. It was concluded that mod3a was the best fit to phase II data. 
Table 4

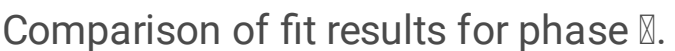

\begin{tabular}{|llll|}
\hline Models & MSE & RMSE & MAE \\
\hline mod1a & 69.2735 & 8.3231 & 5.383 \\
\hline mod2a & 117.1521 & 10.8237 & 5.9 \\
\hline mod3a & 67.7729 & 8.2324 & 5.3772 \\
\hline
\end{tabular}

We used the OvAge calculator from the literature and the established mod3a formula to test the phase I and phase II datasets to compare the chronological age with the predicted ovarian age (Table 5). The Mod3a calculation results show that there was no significant difference between the chronological age of healthy women and the ovarian age predicted by OvAge, and P2 was less than P1, indicating that the difference between the predicted results for healthy people was more significant than that of the OvAge calculator in the literature. The chronological age of PCOS patients was not significantly different from the ovarian age calculated by OvAge $(p>0.05)$. The chronological age of DOR patients and the ovarian age calculated by OvAge were also not significantly different $(p>0.05)$. This finding shows that the accuracy of Mod3a's prediction of ovarian age was worse than that of the literature OvAge calculator.

Table 5

Comparison of chronological age, OvAge calculated age in the literature, and the established mod3a calculated age.

\begin{tabular}{|llllll|}
\hline Indicators & $\begin{array}{l}\text { chronological } \\
\text { age }\end{array}$ & $\begin{array}{l}\text { Literature } \\
\text { OvAge }\end{array}$ & mod3a & $\begin{array}{l}\text { P1 value } \\
\text { (literature) }\end{array}$ & $\begin{array}{l}\text { P2 value } \\
\text { (mod3a) }\end{array}$ \\
\hline $\begin{array}{l}\text { Healthy women }(n= \\
68)\end{array}$ & $30.79 \pm 5.91$ & $31.45 \pm 4.89$ & $\begin{array}{l}31.44 \pm \\
2.27\end{array}$ & 0.3984744 & 0.3344457 \\
\hline DOR women $(n=52)$ & $29.27 \pm 3.76$ & $31.29 \pm 5.42$ & $\begin{array}{l}28.17 \pm \\
2.42\end{array}$ & 0.0402479 & 0.1097118 \\
\hline $\begin{array}{l}\text { PCOS women }(n= \\
\text { 45) }\end{array}$ & $28.33 \pm 2.83$ & $26.55 \pm 2.46$ & $28.66 \pm 1.6$ & 0.0002038 & 0.49838 \\
\hline
\end{tabular}

\section{Optimization of the generalized linear model of the OvAge calculator}

To further optimize the OvAge calculation formula we established by expanding the sample size of the test data, we tentatively developed an online OvAge calculator based on the WeChat applet using the mod3a formula (Fig. 2). With the help of the online OvAge calculator in the WeChat applet, $9000+$ sample data were collected in only two weeks, and data from 97 healthy people were screened out as model optimization dataset III.

As mentioned earlier, we used the data from phase II and phase III, and based on mod1a, mod2a, and mod3a, the models were optimized to mod $1 b, \bmod 2 b$, and mod3b ( $b$ means the selected data came from the sum of phase II data and phase III data). We corrected the differences between datasets from different sources by introducing a new variable New (New $=0$ represents phase II data, New $=1$ represents phase III 
data) and considered adding new variables and their corresponding interaction terms with other variables to this model. However, the results of variable selection based on the stepwise linear regression of the AIC value showed that no significant variable effects related to the new variables were found, which means that there was no difference in AMH and PRL between the phase II and phase III data(Table 6). At the same time, the prediction errors of MSE, RMSE, and MAE were the lowest in mod2b (Table 7), indicating that mod2b was the best formula after the second optimization. The model was estimated as:

OvAge $=\exp (3.5254-0.001 * P R L-0.0231 * A M H)$

Table 6

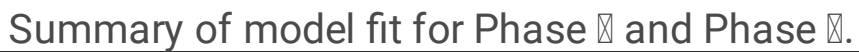

\begin{tabular}{|c|c|c|c|c|c|}
\hline item & & Estimate & Std. Error & t value & $p$ value \\
\hline \multirow[t]{5}{*}{$\bmod 1 b$} & (Intercept) & 33.8402 & 0.8489 & 39.8635 & 0 \\
\hline & PRL & -0.0016 & 0.0072 & -0.2253 & 0.8221 \\
\hline & & & & & $(-)$ \\
\hline & AMH & -0.7045 & 0.1968 & -3.5794 & 0.0005 \\
\hline & & & & & $(\star \star \star)$ \\
\hline \multirow[t]{5}{*}{$\bmod 2 b$} & (Intercept) & 3.5254 & 0.0264 & 133.3673 & 0 \\
\hline & PRL & -0.0001 & 0.0002 & -0.2282 & 0.8195 \\
\hline & & & & & $(-)$ \\
\hline & $\mathrm{AMH}$ & -0.0231 & 0.0064 & -3.6403 & 0.0003 \\
\hline & & & & & $(\star \star \star)$ \\
\hline \multirow[t]{5}{*}{$\bmod 3 b$} & (Intercept) & 33.6966 & 0.8367 & 40.2746 & 0 \\
\hline & PRL & -0.0017 & 0.0071 & -0.2407 & 0.8098 \\
\hline & & & & & $(-)$ \\
\hline & AMH & -0.6608 & 0.1872 & -3.5297 & 0.0004 \\
\hline & & & & & $(\star \star \star)$ \\
\hline
\end{tabular}

Table 7

Evaluation of model fit for Phase $₫$ and

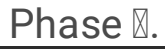

\begin{tabular}{|llll|}
\hline Models & MSE & RMSE & MAE \\
\hline mod1b & 32.0149 & 5.6582 & 4.1787 \\
\hline mod2b & 31.7237 & 5.6324 & 4.1703 \\
\hline mod3b & 32.6351 & 5.7127 & 4.2063 \\
\hline
\end{tabular}


We used the literature formula, the best formula from the first optimization (mod3a), and the best formula from the second optimization (mod2b) to test with the phase I + II dataset (Table 8). The test results show that for the disease population (DOR/PCOS), P1ロP3ロP2, and the accuracy of the calculation results of the ovarian formula after two optimizations was not as good as that of the literature formula, but the accuracy of the calculation results of the second optimization formula was significantly improved. At the same time, for healthy people, $\mathrm{P} 3>\mathrm{P} 1>\mathrm{P} 2$, the calculation results of the first optimization formula were not as accurate as the literature formula, but after the second optimization, the accuracy of the calculation result was better than the literature formula. Mod2b had higher accuracy in predicting the ovarian age of the normal population, which was better than Mod3a and literature formulas. At the same time, the accuracy of predicting the ovarian age of the disease population was better than mod3a, but it was still not as good as the literature formula.

Table 8

Comparison of chronological age and three different models' calculated age.

\begin{tabular}{|llllllll|}
\hline Indicators & $\begin{array}{l}\text { chronological } \\
\text { age }\end{array}$ & $\begin{array}{l}\text { Literature } \\
\text { OvAge }\end{array}$ & mod3a & mod2b & $\begin{array}{l}\text { P1 value } \\
\text { (Literature) }\end{array}$ & $\begin{array}{l}\text { P2 value } \\
\text { (mod3a) }\end{array}$ & $\begin{array}{l}\text { P3 value } \\
\text { (mod2b) }\end{array}$ \\
\hline $\begin{array}{l}\text { Healthy } \\
\text { women } \\
(n=68)\end{array}$ & $30.79 \pm 5.91$ & $\begin{array}{l}31.45 \pm \\
4.89\end{array}$ & $\begin{array}{l}31.44 \\
\pm 2.27\end{array}$ & $\begin{array}{l}30.95 \\
\pm 1.63\end{array}$ & 0.3984744 & 0.3344457 & 0.8169367 \\
\hline $\begin{array}{l}\text { DOR } \\
\text { Women } \\
(n=52)\end{array}$ & $29.27 \pm 3.76$ & $\begin{array}{l}31.29 \pm \\
5.42\end{array}$ & $\begin{array}{l}28.17 \\
\pm 2.42\end{array}$ & $\begin{array}{l}28.28 \\
\pm 1.74\end{array}$ & 0.0402479 & 0.1097118 & 0.1068514 \\
\hline $\begin{array}{l}\text { PCOS } \\
\text { women } \\
(n=45)\end{array}$ & $28.33 \pm 2.83$ & $26.55 \pm$ & 28.66 & 27.63 & 0.0002038 & 0.49838 & 0.4089016 \\
\hline
\end{tabular}

\section{Discussion}

The age of human organs is not exactly the same as the person's chronological age, as is the case for ovaries $^{20-21}$ The reproductive aging process for women is related to factors such as age, genes, environment, stress, diet, and pathology. Therefore, the reproductive system of women of the same age can be different. In view of the differences, how to intuitively and accurately assess women's ovarian reserve, fertility, and menopause is worth considering. At present, there is no satisfactory model that can evaluate the status of the female reproductive system through a single index or a combined index. The ideal model should be able to integrate all relevant information to evaluate ovarian reserve qualitatively and quantitatively. In 2015, the "Ovage" proposed by Ventureira and others met the current requirements. In previous studies, we found that in PCOS and DOR populations, the ovarian age calculated by the existing OvAge model was significantly smaller or greater than its actual age. For healthy people, there was no significant difference between the ovarian age calculated by the OvAge model and actual age, which can reflect ovarian function and potential fertility of these different populations to a certain extent. However, due to its limitations in terms of geography, population, and sample size, this formula may not be fully 
applicable to Chinese women. Therefore, we wanted to establish an ovarian age calculation formula suitable for Chinese women.

Building an accurate scientific model that can quickly predict the age of the ovaries requires a large amount of sample data from subjects. The traditional methods of recruiting subjects mainly include research center recruitment, hospital department recruitment, and third-party CRO company cooperative recruitment. Usually, research centers or hospital departments must recruit subjects themselves, and therefore, it takes a long time to collect a large amount of sample data. Cooperation with third-party CRO companies is also very expensive in addition to being time-consuming. Moreover, $\mathrm{CRO}$ companies with a small scale, lack of influence, low visibility, and weak overall strength cannot achieve nationwide recruitment ${ }^{22}$.

To overcome the shortcomings of traditional recruitment methods, we creatively developed an online OvAge calculator based on WeChat applets, which are widely used in China, to provide a reference value for ovarian age for the Chinese population. This research is also the first application of IOT technology to explore the possibility of a new type of self-report system for Chinese families. We combined the WeChat applet platform and the OvAge calculator by Ventureira. et al. to optimize a new online calculator. This online calculator can collect a large number of samples in a short time, which saves considerable time and greatly reduces the capital cost. At the same time, background operators can use the collected data to continuously optimize and iterate formulas. The subject's self-report system only needs to enter part of the values from the physical examination report, fill out a simple questionnaire, and sign the informed consent form, and then they can obtain their own estimated ovarian age and targeted fertility guidance. It not only ensures a wide range of subjects but also significantly improves the efficiency of the recruitment process and saves the cost of clinical recruitment. For the phase I data in this study, through hospital recruitment, we recruited 113 subjects (16 healthy individuals, 45 PCOS, and 52 DOR populations) within two months. For Phase II data, we collected data on 52 healthy women in two months through cooperation with a CRO recruitment company at a cost of 52,000 yuan. For Phase III data, we used the online OvAge calculator of the WeChat applet to collect a total of more than 9,000 people within two weeks and obtained data on 97 healthy women after screening. In addition, the system is managed by authorized staff, and the network used is located in the hospital network environment and is specially encrypted.

Increasing evidence has shown that the construction of a predictive model of ovarian function based on healthy women's ovarian reserve is of great significance for the accurate assessment of ovarian reserve. There are significant ethnic differences in ovarian reserve and reproductive aging. Blacks, Hispanics, and Asians have significantly lower pregnancy and birth rates than Whites. This large-scale prospective study based on the Chinese population is of great significance for establishing an ovarian age prediction model based on the characteristics of Chinese women. Compared with previous research results, this research not only optimized the OvAge model but also added family history, nutritional status, psychological factors, body mass index, and other indicators that are highly related to fertility (Fig. 2). We used the WeChat applet OvAge calculator to collect a large amount of sample data to continuously optimize the OvAge model, and at the same time, we continued to update the subject's self-report system. Through model optimization, for the Chinese population, we deleted some analysis indicators to make the evaluation results for ovarian age more reliable. However, the online OvAge calculator also has some limitations. For example, subjects must 
obtain a physical examination report before entering a value, which causes some subjects to withdraw from the program when they find that there is no corresponding report. In addition, since the data are input by the subject autonomously, the authenticity of the data needs to be further determined. In the future, we will add image recognition functions and require subjects to upload hospital test reports, and then the computer will extract the values to increase the authenticity of the data. At the same time, our second optimized ovarian age calculation formula, mod2b, is more accurate than the first optimization formula, mod3a, for the three populations, and the accuracy of the ovarian age evaluation results for healthy people is also better than the literature OvAge formula. However, the accuracy of the prediction results for ovarian age for the PCOS and DOR populations is significantly worse than that of the literature OvAge formula. The possible reason for this phenomenon is that the training data (phase II \& phase III) used in this study from the first optimization to the second optimization are only data from healthy people and do not include data from PCOS and DOR populations. In the future, with the continuous promotion of small programs, a large amount of healthy and nonhealthy population data will continue to be incorporated into the back-end system. As of the deadline for manuscript writing (August 26, 2021), the back-end system collected 28,616 samples from the population (Fig. 1). We will continue to use newly collected population data to optimize the existing ovarian age calculation formula. In the near future, with the continuous optimization of the formula, we are confident in establishing a more scientific ovarian age assessment system based on the Chinese population.

In summary, the ovarian age calculator established using data from Chinese population samples can help Chinese women detect changes in ovarian reserve. In addition, it can also intervene in time to improve reproductive potential, predict the time of menopause, improve reproductive prognosis, and increase the success rate of assisted reproductive technology.

\section{Conclusion}

This research combines the existing OvAge model to develop an OvAge online calculator based on the WeChat applet. Then, we collected basic data and gynecological examination reports from Chinese women through an online application, aiming to explore new methods for subject self-report and provide more data support for further optimization of the OvAge model. This approach provides more support for the development and promotion of the online OvAge calculator, creates a new method of network-based recruitment of clinical subjects, and provides new ideas for clinical research.

\section{Abbreviations}

OvAge: Ovarian age;

AMH: Anti-Müllerian hormone;

AFC: Antral follicle counting;

FSH: Follicle-stimulating hormone; 
LH: Luteinizing hormone;

PRL: Prolactin;

$\mathrm{E}_{2}$ : Estradiol;

T: Testosterone;

P: Progesterone;

PCOS: Polycystic ovary syndrome;

DOR: Diminished ovarian reserve;

CRO: Contract research organization

GLM: generalized linear model theory

AIC: Akaike information criterion

BIC: Bayesian information criterion

\section{Declarations}

\section{Availability of data and materials}

Data are not publicly available due to ethical restrictions. Data that support the findings of this study are available from the corresponding author, upon reasonable request and with necessary ethics approval.

\section{Acknowledgments}

This work was supported by grants from Jiangsu Provincial Administration of Traditional Chinese Medicine (ZD201915, a major project of Jiangsu Province's traditional Chinese medicine science and technology development plan in 2019).

\section{Funding}

This research was funded by the Jiangsu Provincial Administration of Traditional Chinese Medicine,grant number: ZD201905.

\section{Author information}

\section{Affiliations}

\section{Department of Histology and Embryology, Nanjing Medical University, Nanjing 211166, China}

Hui Wang, LinTing Han,XueLi Zhao, PanPan Chen\&Haiyang Zhao 
Department of Gynecology, Jiangsu Provincial Hospital of Traditional Chinese Medicine, Affiliated Hospital of Nanjing University of Chinese Medicine, Nanjing 210029, China

Wenwen Xu,Jing Jin\&Qingling Ren

School of Pediatrics, Nanjing Medical University, Nanjing 211166, China

Zheng Zhu

Department of Biostatistics, School of Public Health, Nanjing Medical University, Nanjing 211166, China

Fang Shao

Experimental Teaching Center of Basic Medicine, Nanjing Medical University, Nanjing 211166, China

Hui Wang

\section{Contributorship}

WWX conceived the study ,Complete the first draft of the article. HYZ ,XLZ,LTH,PPC,JJ ,ZZ,Modify the article format and Responsible for collecting data.FS ,QLR,HW Suggest revisions to the article ,they are contributed equally and All authors reviewed the manuscript.

\section{Corresponding author}

Correspondence to Qingling Ren.

\section{Ethics declarations}

\section{Ethical approval and consent to participate}

The study was approved by Chinese Clinical Trial Registry (registration No. ChiCTR2000037522) and Medical ethics committee of Jiangsu Province Hospital of Chinese Medicine (approved No. 2019NL-15202). The subjects were informed of the objectives and modalities of the study and informed consent was obtained,All collected data was in accordance to guidelines of Declaration of Helsinki in the manuscript.

\section{Consent for publication}

Not applicable.

\section{Competing interests}

The authors declare no conflict of interest.

\section{References}


1. Soules MR, Sherman S, Parrott E, et al. Executive summary: Stages of Reproductive Aging Workshop (STRAW). Climacteric: the journal of the International Menopause Society. 2001;4(4):267-272.

2. Harlow SD, Gass M, Hall JE, et al. Executive summary of the Stages of Reproductive Aging Workshop + 10: addressing the unfinished agenda of staging reproductive aging. The Journal of clinical endocrinology and metabolism. 2012;97(4):1159-1168.

3. Venturella R, Lico D, Sarica A, et al. OvAge: a new methodology to quantify ovarian reserve combining clinical, biochemical and 3D-ultrasonographic parameters. Journal of ovarian research. 2015;8:21.

4. Kushnir VA, Barad DH, Gleicher N. Ovarian reserve screening before contraception? Reproductive biomedicine online. 2014;29(5):527-529.

5. Xu H, Shi L, Feng G, et al. An Ovarian Reserve Assessment Model Based on Anti-Müllerian Hormone Levels, Follicle-Stimulating Hormone Levels, and Age: Retrospective Cohort Study. Journal of medical Internet research. 2020;22(9):e19096.

6. Tal R, Seifer DB. Potential mechanisms for racial and ethnic differences in antimüllerian hormone and ovarian reserve. International journal of endocrinology. 2013;2013:818912.

7. Faddy MJ, Gosden RG, Gougeon A, et al. Accelerated disappearance of ovarian follicles in mid-life: implications for forecasting menopause. Human reproduction (Oxford, England). 1992;7(10):13421346.

8. von Meyenn F, Reik W. Forget the Parents: Epigenetic Reprogramming in Human Germ Cells. Cell. 2015;161(6):1248-1251.

9. Haruty B, Friedman J, Hopp S, et al. Reproductive health and the environment: Counseling patients about risks. Cleveland Clinic journal of medicine. 2016;83(5):367-372.

10. Hawkins Bressler L, Bernardi LA, De Chavez PJ, et al. Alcohol, cigarette smoking, and ovarian reserve in reproductive-age African-American women. American journal of obstetrics and gynecology. 2016;215(6):758.e1-.e9.

11. Lambertini M, Del Mastro L, Pescio MC, et al. Peccatori FA, et al. Cancer and fertility preservation: international recommendations from an expert meeting. BMC medicine. 2016;14:1.

12. Tal R, Seifer DB. Ovarian reserve testing: a user's guide. American journal of obstetrics and gynecology. 2017;217(2):129-140.

13. Testing and interpreting measures of ovarian reserve: a committee opinion. Fertility and sterility. 2020;114(6):1151-1157.

14. Lew R. Natural history of ovarian function including assessment of ovarian reserve and premature ovarian failure. Best practice \& research Clinical obstetrics \& gynaecology. 2019;55:2-13.

15. Kučera M, Štěpán MI J, Štelcl M. Possibilities and real meaning of assessment of ovarian reserve. Ceska gynekologie. 2018;83(4):307-311.

16. Iwase A, Osuka S, Goto M, et al. Clinical application of serum anti-Müllerian hormone as an ovarian reserve marker: A review of recent studies. The journal of obstetrics and gynaecology research. 2018;44(6):998-1006. 
17. Shao F, He Z, Zhu Z, et al. Internet Influence of Assisted Reproduction Technology Centers in China: Qualitative Study Based on WeChat Official Accounts. Journal of medical Internet research. 2020;22(6):e17997.

18. Revised 2003 consensus on diagnostic criteria and long-term health risks related to polycystic ovary syndrome (PCOS). Human reproduction (Oxford, England). 2004;19(1):41-47.

19. Kawwass JF, Hipp HS, Session DR, et al. Severity of Diminished Ovarian Reserve and Chance of Success with Assisted Reproductive Technology. The Journal of reproductive medicine. 2017;62(34):153-160.

20. Ori A, Toyama BH, Harris MS, et al. Integrated Transcriptome and Proteome Analyses Reveal OrganSpecific Proteome Deterioration in Old Rats. Cell systems. 2015;1(3):224-237.

21. Bonner C, Bell K, Jansen J, et al. Should heart age calculators be used alongside absolute cardiovascular disease risk assessment? BMC cardiovascular disorders. 2018;18(1):19.

22. Shuchman M. Commercializing clinical trials-risks and benefits of the CRO boom. The New England journal of medicine. 2007;357(14):1365-1368.

\section{Figures}


Definition and creation of three datasets (training set, testing set, prediction set)

Descriptive statistics

Model construction based on the training set

Choice of the best model by performances on the testing set

Evaluate the final model by application on the prediction set

Figure 1

Flow chart of optimizing the OvAge model 


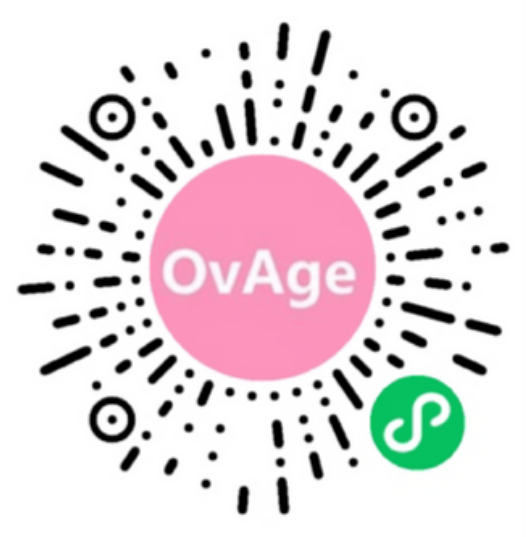

a
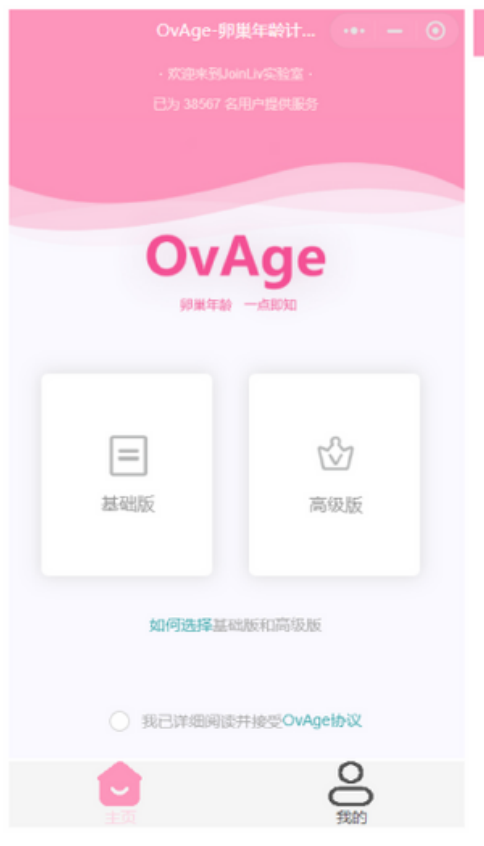

b

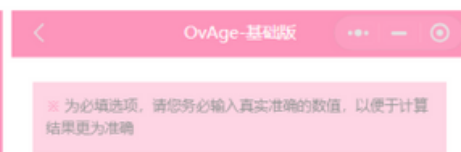

1. 年龄 (岁)

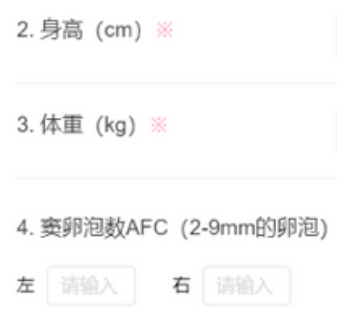

5. 人抗苗勒试管激索AMH

5.

$\mathrm{ng} / \mathrm{mL} \bigcirc \mathrm{pmol} / \mathrm{L} \bigcirc$ 其他

C
1. 怀疋次数

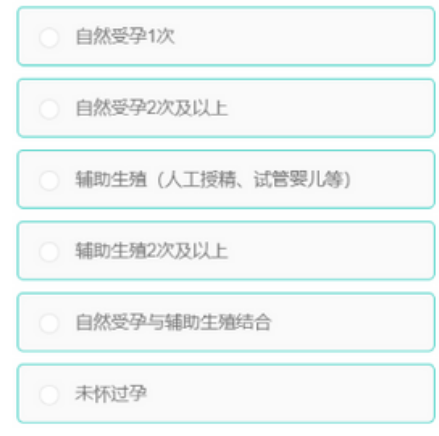

2. 产子数

d

Figure 2

A subject self-report system based on the WeChat applet-online OvAge calculator.a.QR code of online

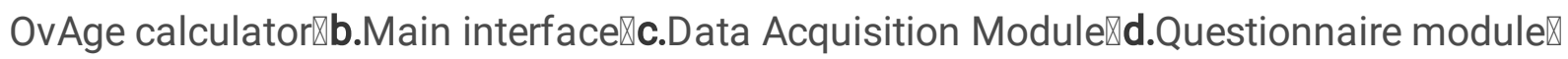

